\title{
FIBRATIONS THAT ARE COFIBRATIONS
}

\author{
JUAN M. ALONSO
}

\begin{abstract}
We give necessary and sufficient conditions for a homotopy cartesian square to be homotopy cocartesian. Specializing, we obtain a necessary and sufficient condition for a fibration to be a cofibration. We apply the above to localization of spaces and to acyclic maps.
\end{abstract}

1. Introduction. R. J. Milgram [5, p. 246] proved that the fibration $K(Q / Z, n) \rightarrow$ $K(Z, n+1) \rightarrow K(Q, n+1)(n \geqslant 1)$ is also a cofibration, and raised the question of which fibrations are also cofibrations. The following answer was recently provided by S. J. Schiffman [9, p. 143], and is also contained-in a more general form-in an earlier paper by Z. Wojtkowiak [10,p. 508]: the fibration (*) $F \rightarrow X \rightarrow X_{P}$ is also a cofibration, where $X$ is a l-connected space (all spaces are assumed to have the homotopy type of $\mathrm{CW}$ complexes), $P$ is a set of primes, $X \rightarrow X_{P}$ is a $P$-localizing map, and $F$ is its homotopy fibre.

We use a classic result of T. Ganea [2, Theorem (1.1)] to find necessary and sufficient conditions for a fibration to be a cofibration (Corollary (2.10)). When applied to the situation described above, we obtain (Corollary (3.2)): if $X$ is nilpotent. then (*) is a cofibration iff $X_{P}$ is 1-connected (or, trivially, $X$ is already $P$-local). Actually, we formulate our results in terms of squares; in this case, Ganea's theorem takes the more symmetric form given by Y. Nomura [8, p. 259] (see also [7, p. 260]).

In $\$ 4$ we deal with the relation between fibrations that are cofibrations and acyclic maps.

We would like to thank Professors I. Berstein and K. S. Brown for helpful conversations, and also thank the referee who pointed out a mistake in an earlier draft of this paper and made helpful suggestions.

2. Squares. See [7] for definitions and properties of homotopy cartesian and homotopy cocartesian squares. Given maps $f: X \rightarrow B$ and $g: Y \rightarrow B$, form the homotopy cartesian square

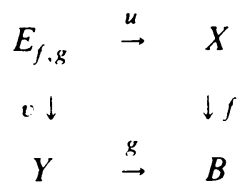

Received by the editors May 28, 1981 and. in revised form. July 12, 1982.

1980 Mathematics Subject Classification. Primary 55P99. 55P60.

. 1983 American Mathematical Societs (00)2-9939/82/0000)-0764;\$(1).(0) 
where $E_{f, g}=\left\{(y, w, x) \in Y \times B^{I} \times X \mid f(x)=w(1)\right.$ and $\left.g(y)=w(0)\right\}$, and $u$ and $v$ are the projections. The square $(2.1)$ is homotopy commutative, with homotopy $H$ : $g \circ v \simeq f \circ v$, given by $H(y, w, x, t)=w(t)$.

From $u$ and $v$ in (2.1) we form the homotopy cocartesian square

$$
\begin{array}{ccc}
E_{f . g} & \stackrel{u}{\rightarrow} & X \\
v \downarrow & & \downarrow i \\
Y & \stackrel{j}{\rightarrow} & C_{u, v}
\end{array}
$$

where $C_{u, v}$ is the double mapping cylinder of $u$ and $v$, and $i, j$ are the inclusions. Then there is a map $r: C_{u, v} \rightarrow B$ (defined using the maps $f, g$ and $H$ ) with $r \circ i=f$ and $r \circ j=g$, that fits in the diagram:

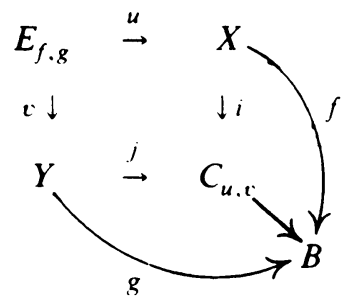

(2.3) Definition. We say that the maps $f$ and $g$ are a bicartesian pair if the map $r$ in (2.2) is a homotopy equivalence (i.e., if (2.1) is both a homotopy cartesian and a homotopy cocartesian square).

(2.4) REMARK. When, say $Y \simeq *, f$ and $g$ are a bicartesian pair iff the fibration $F_{f} \rightarrow X \stackrel{f}{\rightarrow} B$ is also a cofibration, where $F_{f}$ is the homotopy fibre of $f$. Notice that in this case $F_{g} \simeq \Omega B$.

(2.5) THEOREM. Let $X, Y$ and $B$ be 0-connected spaces, and let $f, g$ and $r$ be as above. Then the following conditions are equivalent:

(i) The maps $f$ and $g$ are a bicartesian pair.

(ii) $F_{r}$ is contractible.

(iii) $F_{r}$ is acyclic (i.e., $\tilde{H}_{*}\left(F_{r}\right)=0$ ).

(iv) There exists a set of primes $P$ such that one of the homologies, $\tilde{H}_{*}\left(F_{f}\right)$ and $\tilde{H}_{*}\left(F_{g}\right)$, is P-torsion and the other is uniquely P-divisible.'

To prove the theorem, we need the following algebraic lemma.

(2.6) Lemma. $A$ pair of abelian groups $A, B$ satisfy $A \otimes B=0=\operatorname{Tor}(A, B)$ if and only if there exists a set $P$ of primes such that one of the groups is $P$-torsion and the other is uniquely $P$-divisible.

Proof. Follows easily from [1, (2.2), (2.3)].

Proof of (2.5). The equivalence of (i) and (ii) follows from Whitehead's theorem, and (ii) $\Rightarrow$ (iii) is trivial.

\footnotetext{
'The equivalence of this condition with (i)-(iii) was suggested by the referee, who also brought Lemma (2.6) and its proof to my attention.
} 
(iii) $\Rightarrow$ (ii). Nomura [8, Theorem (3.4)] (see also [7, Theorem 47]) has shown that the fibre $F_{r}$ of $r$ is homotopy equivalent to the join $F_{f} * F_{g}$ of the fibres of $f$ and $g$. The homology of this join is [6]

$$
\tilde{H}_{t+1}\left(F_{f} * F_{g}\right)=\bigoplus_{i+j=t} \tilde{H}_{i}\left(F_{f}\right) \otimes \tilde{H}_{j}\left(F_{g}\right) \oplus \bigoplus_{i+j=t-1} \operatorname{Tor}\left(\tilde{H}_{i}\left(F_{f}\right), \tilde{H}_{j}\left(F_{g}\right)\right)
$$

In particular, $0=\tilde{H}_{1}\left(F_{r}\right)=\tilde{H}_{0}\left(F_{f}\right) \otimes \tilde{H}_{0}\left(F_{g}\right)$. Since $\tilde{H}_{0}(-)$ is either trivial or free abelian, either $F_{f}$ or $F_{g}$ must be 0 -connected. Then $F_{f} * F_{g}$ is 1 -connected [6], and being acyclic, it is contractible.

(iii) $\Leftrightarrow$ (iv) follows from (2.7) and Lemma (2.6) applied to $A=\bigoplus_{i} \tilde{H}_{i}\left(F_{f}\right)$ and $B=\bigoplus_{j} \tilde{H}_{j}\left(F_{g}\right)$.

(2.8) Remark. When $P$ is empty, condition (iv) means that one of the fibres is acyclic.

(2.9) COROLLARY. With the same hypothesis of Theorem (2.5), we have:

(a) If one of $F_{f}$ or $F_{g}$ is acyclic, then $f$ and $g$ are a bicartesian pair.

(b) If $F_{f}$ is not 0-connected, then $f$ and $g$ are a bicartesian pair if and only if $F_{g}$ is acyclic.

Proof. (a) follows from (2.8), and (b) from the fact that $\tilde{H}_{*}\left(F_{f}\right)$ satisfies (iv) in (2.5) if and only if $P$ is empty.

For the case of a fibration (see (2.4)), we have

(2.10) Corollary. Let $(*) F_{f} \rightarrow X \stackrel{f}{\rightarrow}$ B be a fibration. Then:

(a) (*) is also a cofibration iff there exists a set of primes $P$ such that one of the homologies, $\tilde{H}_{*}\left(F_{f}\right)$ and $\tilde{H}_{*}(\Omega B)$, is $P$-torsion and the other is uniquely $P$-divisible.

(b) If $F_{f}$ is acyclic, then $(*)$ is also a cofibration.

(c) If $\pi_{1}(B) \neq 0$, then $(*)$ is also a cofibration iff $F_{f}$ is acyclic.

3. Localization. We apply the above to the following situation: let $P$ be a set of primes, $X$ and $Y$ nilpotent spaces (see [4]); $B=X_{P}$, the $P$-localization of $X$, and $f$ : $X \rightarrow X_{P}$ is a $P$-localizing map. We also assume that $Y$ is a $P$-local space; $g: Y \rightarrow X_{P}$ is any map.

(3.1) Proposition. The maps $f$ and $g$ are a bicartesian pair iff either both $F_{f}$ and $F_{g}$ are 0-connected, or at least one of these fibres is acyclic.

(3.2) COROLlaRY. The fibration $F_{f} \rightarrow X \stackrel{f}{\rightarrow} X_{P}$ is also a cofibration iff either $X_{P}$ is 1-connected, or $X$ is P-local.

Proof of (3.2). In view of (2.4) and (3.1), we need only show that $F_{f}$ acyclic implies that $X$ is $P$-local.

The fibre $F_{f}$ is a nilpotent space [4, p. 62], so that $\pi_{1}\left(F_{f}\right)$ is a nilpotent, perfect group ( $F_{f}$ acyclic); thus it is trivial, and $F_{f}$ is a 1 -connected acyclic space, hence contractible. Then $f$ is a homotopy equivalence, showing that $X$ is $P$-local.

Proof of (3.1). $(\Rightarrow)$ This follows from (2.9)(b).

$(\Leftarrow)$ If one of the fibres is acyclic, use (2.9)(a). So assume that both fibres are 0 -connected; then $F_{f}$ and $F_{g}$ are nilpotent spaces and we claim that $F_{g} \simeq\left(F_{g}\right)_{P}$ and 
$\left(F_{f}\right)_{P}=*$. This implies that $\tilde{H}_{*}\left(F_{f}\right)$ is $P^{\prime}$-torsion and that $\tilde{H}_{*}\left(F_{g}\right)$ is uniquely $P^{\prime}$-divisible, where $P^{\prime}$ is the set of primes complementary to $P$. Now use (2.5)(iv).

To complete the proof, consider the homotopy commutative diagrams:

$$
\begin{array}{cccccccccc}
F_{g} & \rightarrow & Y & \stackrel{g}{\rightarrow} & X_{P} & F_{f} & \rightarrow & X & \rightarrow & X_{P} \\
\downarrow & & \downarrow & & \downarrow \text { id } & \downarrow & & \downarrow f & & \text { lid } \\
\left(F_{g}\right)_{P} & \rightarrow & Y_{P} & \rightarrow & X_{P} & \left(F_{f}\right)_{P} & \rightarrow & X_{P} & \rightarrow & X_{P}
\end{array}
$$

Both are obtained from the fibrations on top by localizing at $P$, and the bottom rows are also fibrations [4, p. 77]. This establishes our claim.

4. Acyclic maps. Hausmann and Husemoller give in [3] several definitions of acyclic maps, and establish many of their properties. We give some other equivalent definitions of acyclicity, and show how these can be used to give different proofs of some of their results.

(4.1) Definition. Let $X$ and $B$ be 0 -connected spaces, and $f: X \rightarrow B$ a map with homotopy fibre (resp.. homotopy cofibre) $F_{f}$ (resp.. $C_{f}$ ). We call $f$ acyclic if $F_{f}$ is an acyclic space.

(4.2) Proposition. Let $X, B$ and $f$ be as above. Then the following conditions are equivalent:

(i) $f$ is acyclic.

(ii) $\Sigma F_{f}$ is contractible.

(iii) The fibration $F_{f} \rightarrow X \stackrel{f}{\rightarrow} B$ is also a cofibration, and $C_{f}$ is contractible.

Moreover, if $\pi_{1}(B)=0$, the above conditions are equivalent to:

(iv) $C_{f}$ is contractible;

and if $\pi_{1}(B) \neq 0$, then they are equivalent to:

(v) The fibration $F_{f} \rightarrow X \stackrel{f}{\rightarrow} B$ is also a cofibration.

Proof. (i) $\Leftrightarrow$ (ii). This is clear, in view of the isomorphism $\tilde{H}_{j}\left(F_{f}\right) \cong \tilde{H}_{j+1}\left(\sum F_{f}\right)$, and the fact that if $F_{f}$ is 0 -connected, then $\Sigma F_{f}$ is 1 -connected.

(i) $\Rightarrow$ (iii). That the fibration is also a cofibration follows from (2.10); but if it is a cofibration, then $C_{f} \simeq \Sigma F_{f} \simeq *$.

(iii) $\Rightarrow$ (ii). From the cofibration $F_{f} \rightarrow X \stackrel{f}{\rightarrow} B$ we obtain that $\Sigma F_{f} \simeq C_{f} \simeq *$.

(iv) $\Rightarrow$ (i). This follows easily from $[3,(1.2)(c)$ or $(1.2)(d)]$.

(v) $\Rightarrow$ (i). This is $(2.10)(\mathrm{c})$.

We use (4.2) to prove the following (cf. [3, Corollary (3.2)])

(4.3) Proposition. Let $A$ be an acyclic space and $f: A \rightarrow Y$ a map with $\pi_{1}(f)=0$. Then $f$ is null-homotopic.

Proof. The condition $\pi_{1}(f)=0$ implies that $f$ lifts to a map $\tilde{f}: A \rightarrow \tilde{Y}$, where $\tilde{Y}$ is the universal cover of $Y$. Consider the cofibration $A \stackrel{\tilde{f}}{\rightarrow} \tilde{Y} \stackrel{u}{\rightarrow} C_{\tilde{f}}$. By Van-Kampen's theorem, $C_{\tilde{f}}$ is 1-connected, and $C_{u} \simeq \sum A \simeq *$, since $A$ is acyclic. By (4.2)(iv), $u$ is an acyclic map. But since both $\tilde{Y}$ and $\tilde{C}_{\tilde{f}}$ are 1-connected, it follows from $[3,(1.5)]$ that 
$u$ is a homotopy equivalence. So that from $u \circ \tilde{f} \simeq *$, we obtain that $\tilde{f} \simeq *$, and this implies that $f$ itself is null-homotopic.

For the next result, cf. [3, Proposition (3.1)].

(4.4) Corollary. Let $f: A \rightarrow Y$ be an acyclic map, and $f^{\prime}: A \rightarrow Y^{\prime}$ be any map. Then there exists a map $h: Y \rightarrow Y^{\prime}$ with $h \circ f \simeq f^{\prime}$ iff $\operatorname{ker} \pi_{1}(f) \subseteq \operatorname{ker} \pi_{1}\left(f^{\prime}\right)$.

Proof. $(\epsilon)$ By (4.2)(iii), the fibration (*) $F_{f} \stackrel{\jmath}{\rightarrow} A \stackrel{f}{\rightarrow} Y$ is also a cofibration. The domain of $\left(f^{\prime} \circ j\right): F_{f} \rightarrow Y^{\prime}$ is an acyclic space, and $\pi_{1}\left(f^{\prime} \circ j\right)=0$. By (4.3). $f^{\prime} \circ j \simeq *$, and since $(*)$ is also a cofibration, it follows that there is a map $h: Y \rightarrow Y^{\prime \prime}$ with $h \circ f \simeq f^{\prime}$.

(4.5) Remark. Proposition (4.3) is obtained in [3] as a corollary of our Corollary (4.4), which in its turn is proved using a result of Quillen (see [3. Proposition (2.3)]).

\section{REFERENCES}

I. A. K. Bousfield, Types of aciclicity. J. Pure Appl. Algebra 4 (1974). 293-298.

2. T. (ianea, A generalization of the homology and homotopy suspension. Comment Math. Helv. 39 (1965), 295-322.

3. J. C. Hausmann and D. Husemoller. Acvelic maps, Enseignement Math. 25 (1979). 53-75.

4. P. J. Hilton. (j. Mislin and J. Roitberg. Localization of nilpotent groups and spaces. Math. Studies. no. 15. North-Holland, Amsterdam. 1975

5. R. J. Milgram, Surgery with coefficients, Ann. of Math. (2) 100 (1974), 194-248

6. J. W. Milnor. Construction of universal hundles. II, Ann. of Math (2) 63 (1956), 430-436.

7. M. Mather, Pull-hacks in homotopy theor, Canad. J. Math. 28 (1976). 225-263

8. Y. Nomura, On extensions of triads. Nagova Math. J. 27 (1966), 249-277.

9. S. J. Schiffman. A mod $p$ Whitehead theorem. Proc. Amer. Math. Soc. 82 (1981), 139-144.

10. Z. Wojtkowiak. On fibrations which are also cofihratıons. Quart. J. Math. Oxford Ser. (2) 30 (1979). $505-512$.

Department of Mathematics. Cornell University. Ithaca. New York 14853

(urrent address: Department of Mathematics. University of Stockholm. S-113 85 Stockholm. Sweden 